\title{
Effect of growth hormone on thin endometrium via intrauterine infusion
}

\author{
Wenjie Li, Zhiwen Cao, Xiaoying Yu, Weihua Hu \\ Center of Reproductive Medicine, the First Affiliated Yijishan Hospital of Wannan Medical College, Wuhu, China \\ Contributions: (I) Conception and design: W Li, W Hu; (II) Administrative support: None; (III) Provision of study materials or patients: None; (IV) \\ Collection and assembly of data: Z Cao; (V) Data analysis and interpretation: Z Cao; (VI) Manuscript writing: All authors; (VII) Final approval of \\ manuscript: All authors. \\ Correspondence to: Weihua Hu. Center of Reproductive Medicine, the First Affiliated Yijishan Hospital of Wannan Medical College, 2 Zhe Shan West \\ Road, Wuhu 241001, China. Email: weihuahu@126.com.
}

\begin{abstract}
Background: A thin endometrium has become a common reason for the repeated implantation failure. Growth hormone $(\mathrm{GH})$ can regulate the proliferation and metabolism of endometrial cells. The aim of this study was to explore the effect of $\mathrm{GH}$ on thin endometrium.

Methods: A total of 48 female Sprague-Dawley (SD) rats were randomly assigned to the following 4 groups with 12 rats in each group: blank control, model, subcutaneous, and GH groups. The blank control group was untreated and maintained in a routine manner. The model, subcutaneous, and GH groups were intrauterine perfused with $95 \%$ ethanol during estrus. After 6-8 h, the model group was intrauterine perfused with $0.2 \mathrm{~mL}$ normal saline, the subcutaneous group received subcutaneous injection of $0.12 \mathrm{mg} / \mathrm{kg}$ $\mathrm{GH}$ dissolved in $0.2 \mathrm{~mL}$ normal saline, and the $\mathrm{GH}$ group was intrauterine perfused with $0.12 \mathrm{mg} / \mathrm{kg} \mathrm{GH}$ dissolved in $0.2 \mathrm{~mL}$ normal saline. Hematoxylin and eosin (HE) staining was used to examine the thickness of the endometrium. The expression of cytokeratin and vimentin was detected by western blotting and immunohistochemistry.
\end{abstract}

Results: The intima thickness in the GH group and blank control group was increased compared with that in the model group $(\mathrm{P}<0.01)$. The intima thickness in the subcutaneous group was increased compared with that in the model group, but there was no significant difference. The expression of vimentin and cytokeratin was increased in the $\mathrm{GH}(\mathrm{P}<0.01)$ and blank control $(\mathrm{P}<0.01)$ groups compared with that in the model group. Conclusions: Intrauterine perfusion of $\mathrm{GH}$ can promote the regeneration and repair of thin endometrium in rats. The therapeutic effect of uterine infusion of GH is better than that of subcutaneous injection of GH.

Keywords: Growth hormone (GH); intrauterine perfusion; thin endometrium; animal model

Submitted Jun 17, 2021. Accepted for publication Aug 11, 2021.

doi: $10.21037 / \mathrm{atm}-21-3583$

View this article at: https://dx.doi.org/10.21037/atm-21-3583

\section{Introduction}

A thin endometrium (1) is generally defined as endometrial thickness below the appropriate threshold, which is usually less than $7 \mathrm{~mm}$. Endometrial thickness is a hallmark of endometrial receptivity and a prognostic factor for implantation (2). A thin endometrium has become a common reason for the frequent cancellation of embryo transfers or repeated implantation failure during assisted reproductive treatment. An increasing number of studies have highlighted the importance of endometrial thickness. For example, some studies have indicated that when the endometrial thickness is greater than $9-10 \mathrm{~mm}$, the clinical pregnancy rate is significantly improved (3). von Wolff et al. (4) showed that the clinical pregnancy rates with endometrial thicknesses $\leq 7$ and $>7 \mathrm{~mm}$ were $7.4 \%$ and $30.8 \%$, respectively, in $225 \mathrm{NC}-\mathrm{IVF}$ cycles.

Infection and intrauterine manipulation are the most common pathogenic factors for a thin endometrium, along 
with age and medication. There are many ways to treat thin endometrium, including aspirin, purified menstrual blood stem cells, pelvic neuromuscular electrical stimulation, sildenafil, stem cells, and colony-stimulating factor $(5,6)$, however, these methods have weak effects. More effective treatments are still needed.

Growth hormone $(\mathrm{GH})$ is a protein and is secreted by the pituitary gland. Previous research has shown that GH mainly regulates the metabolism of human substances. Recently, GH has been shown to play an important role in the reproductive system. Studies have shown that GH is expressed in decidual tissue cells and late endometrial cells during early pregnancy (7). Other studies have noted (8) that the proliferation and metabolism of endometrial cells can be regulated by GH. Insulin-like growth factors (IGF) distributed on endometrial cells are bound by GH and it acts locally in the endometrium via paracrine and autocrine mechanisms. Cui et al. (9) performed a clinical study in combination with in vitro experiment to clarify the effect of GH. Patients treated with GH had a significantly greater endometrium thickness on day 3 compared with the control group $(\mathrm{P}<0.05)$. A dose-dependent increase of viability in the RL95-2 cells was observed while they were incubated with GH in CCK-8 assay. Based on the successful construction of rats with thin endometria, we studied the effect of GH intrauterine perfusion on thin endometrium. We present the following article in accordance with the ARRIVE reporting checklist (available at https://dx.doi. org/10.21037/atm-21-3583).

\section{Methods}

\section{Animals}

A total of 48 female Sprague-Dawley (SD) rats, weighing $230 \pm 15 \mathrm{~g}$ and aged $8-10$ weeks, were used in this study. The experimental rats were recruited from the Medical Animal Experimental Center of Nanjing Medical University [Rat Animal License: SCXK (Su) 2016-0002]. The rats were maintained in the specific-pathogen free (SPF) animal room of Wannan Medical College under a $12 \mathrm{~h}$ light/dark cycle with ad libitum access to water and food at a relative humidity of $45-65 \%$ and an ambient temperature of $24 \pm 2{ }^{\circ} \mathrm{C}$. Rat vaginal smears were performed daily between 8-9 am and 5-6 pm. Rats with 3 complete estrous cycles were chosen for the experiment. Experiments were performed under a project license (No.: LLSC-2019-001) granted by the First Affiliated Yijishan Hospital of Wannan
Medical College Ethics Committee. All animal experiments are carried out in accordance with the National Institutes of Health Guide for the Care and Use of Laboratory animals.

\section{Reagents and instruments}

A volume fraction of $95 \%$ alcohol was purchased from Shandong Anjie Gaoke Technology Co., Ltd. (Shandong, China). The GH was purchased from Anhui Anke Bio-Tech Co., Ltd. (Hefei, Anhui, China). The rabbit anti-cytokeratin antibody was purchased from Novus Biologicals (Littleton, CO, USA), and the rabbit anti-vimentin antibody was purchased from Cell Signaling Technology (Danvers, MA, USA). The immunohistochemistry (IHC) kit was purchased from Beijing Zhongshang Jinqiao Co., Ltd. (Beijing, China) and the microscope was purchased from Olympus Corp. (Shinjuku, Tokyo, Japan).

\section{Model preparation}

A daily vaginal smear was used to examine estrus in rats. The rats with stable estrus were injected intraperitoneally with $10 \%$ chloral hydrate $(0.3 \mathrm{~mL} / 100 \mathrm{~g})$ during estrus (10). The skin in the abdominal surgical area was prepared, the rat was placed on a sterile operating table, and the skin was disinfected with iodophor 3 times. Then, $2-2.5 \mathrm{~cm}$ above the pubic symphysis, a longitudinal opening of the abdomen was cut to $2-3 \mathrm{~cm}$, and the abdomen was opened layer-bylayer. One side of the uterus was ligated near the vagina and ovary. We then injected $0.2 \mathrm{~mL} 95 \%$ ethanol into the uterus with a syringe, with the syringe kept in place for $5 \mathrm{~min}$. Then, the remaining ethanol was pumped back out, and physiological saline was utilized to flush the uterine cavity twice. It was confirmed that there was no continuous bleeding at the incision site and that the uterus had returned to the normal anatomical position. The incision was sutured layer-by-layer, and the incision area was disinfected with iodophor 3 times.

\section{Grouping method and processing}

A total of 48 female rats were randomly assigned to the following 4 groups with 12 rats in each group: blank control, model, subcutaneous, and GH groups. The blank control group was maintained normally without intervention. The model, subcutaneous, and GH groups were intrauterine perfused with $95 \%$ ethanol during estrus. After 6-8 h, the model group was intrauterine perfused with 
$0.2 \mathrm{~mL}$ normal saline, the subcutaneous group received a subcutaneous injection of $0.12 \mathrm{mg} / \mathrm{kg}$ GH dissolved in $0.2 \mathrm{~mL}$ normal saline, and the $\mathrm{GH}$ group was intrauterine perfused with $0.12 \mathrm{mg} / \mathrm{kg}$ GH dissolved in $0.2 \mathrm{~mL}$ normal saline.

\section{Detection indicators and methods}

After 3 estrous cycles (12-15 days), the rats were sacrificed during estrus. The uterus was placed in $10 \%$ neutral formalin for 24-48 h, dehydrated, and embedded in paraffin. Hematoxylin and eosin (HE) staining was performed to determine the thickness of the endometrium. We used IHC and western blotting to verify the expression of endometrial cytokeratin and vimentin.

\section{HE staining}

The rat uterus was placed in a wax block and sliced at a thickness of $5 \mu \mathrm{m}$. The average thickness was obtained with the Leica Qwin Plus image processing software (Leica Microsystems Inc., Buffalo, IL, USA), and the vertical distance from the uterine cavity to the myometrium was measured (5 fields of view were randomly obtained).

\section{Detection of cytokeratin and vimentin by IHC}

The expression of cytokeratin and vimentin was compared by semi-quantitative method. A total of 7 visual fields were randomly selected under high magnification mirror $(\times 400)$, and the average optical density was calculated by Image J (National Institutes of Health, Bethesda, MD, USA). The average optical density (mean optical density, MOD) = cumulative optical density (integrated optical density, IOD)/ area.

The paraffin specimens were sliced and dewaxed in a $60{ }^{\circ} \mathrm{C}$ oven for $120 \mathrm{~min}$ and incubated with xylene I and II for $5 \mathrm{~min}$. Then, the slides were subjected to $100 \%$ ethanol immunodehydration, routine dehydration for 5 minutes, ethylenediamine tetraacetic acid (EDTA) hightemperature antigen retrieval for $3 \mathrm{~min}$, natural cooling for $10 \mathrm{~min}$, a cold-water soak for $10 \mathrm{~min}$, a complete soak in $3 \%$ hydrogen peroxide for $10 \mathrm{~min}$, and 3 phosphatebuffered saline (PBS) rinses for $5 \mathrm{~min}$ each. Then, a ring was drawn with an IHC pen. The slides were sealed with $5 \%$ bovine serum albumin (BSA) at room temperature for $1 \mathrm{~h}$, the BSA solution was discarded, and the remaining solution was removed by pipetting. The tissues were incubated with diluted primary antibody overnight at $4{ }^{\circ} \mathrm{C}$. The primary antibody was removed, and the sample was rinsed with PBS and incubated with secondary antibody for $1 \mathrm{~h}$ at room temperature. After color development with 3,3'-diaminobenzidine (DAB), the sample was counterstained for 2-3 min, developed for $30 \mathrm{~s}$, rinsed with water for $2 \mathrm{~min}$, returned to room temperature for $2 \mathrm{~min}$, incubated with $100 \%$ ethanol, dehydrated for $2 \mathrm{~min}$, blown dry, and sealed with a neutral resin. A high magnification $(\times 400)$ image was taken under a microscope. The results were quantified with Image-Pro Plus 6.0 software (Media Cybernetics, Rockville, MD, USA).

\section{Western blot analysis of protein expression}

The uterine specimen was obtained from $-80{ }^{\circ} \mathrm{C}$ storage and quickly scraped off on ice, then transferred to a centrifuge tube. The tissue $[300 \mu \mathrm{L}$ radioimmunoprecipitation assay (RIPA)] was homogenized on ice for $30 \mathrm{~min}$, then centrifuged to remove the supernatant. In total, $100 \mu \mathrm{L}$ supernatant were added to $100 \mu \mathrm{L} 5 \times$ loading buffer and incubated in a boiling water bath for $5 \mathrm{~min}$. The sample was resolved by electrophoresis, transferred to a membrane, blocked with milk for $2 \mathrm{~h}$, washed for $5 \mathrm{~min}$, incubated with the appropriate primary antibody $(1: 1,000)$, and shaken at $4{ }^{\circ} \mathrm{C}$ overnight. The membrane was washed with Trisbuffered saline with Tween 20 (TBST) for 5 min 3 times and incubated with a secondary antibody diluted in TBST $(1: 5,000)$ at room temperature for $2 \mathrm{~h}$. Then, the membrane was washed, developing agent was added dropwise, and the membrane was exposed to film. Image J was used to measure and compare the expression of the target protein.

\section{Statistical analysis}

The statistical software SPSS 22.0 (IBM Corp., Chicago, IL, USA) was used to perform the analysis. The measured data were expressed as mean \pm standard deviation. Comparisons were made among the groups by a one-way analysis of variance (ANOVA). Statistical significance was indicated by $\mathrm{a}=0.05$ and $\mathrm{P}<0.05$.

\section{Results}

\section{HE results}

The morphology of the uterus in the blank control group was intact, and the interstitial space was narrow. The 

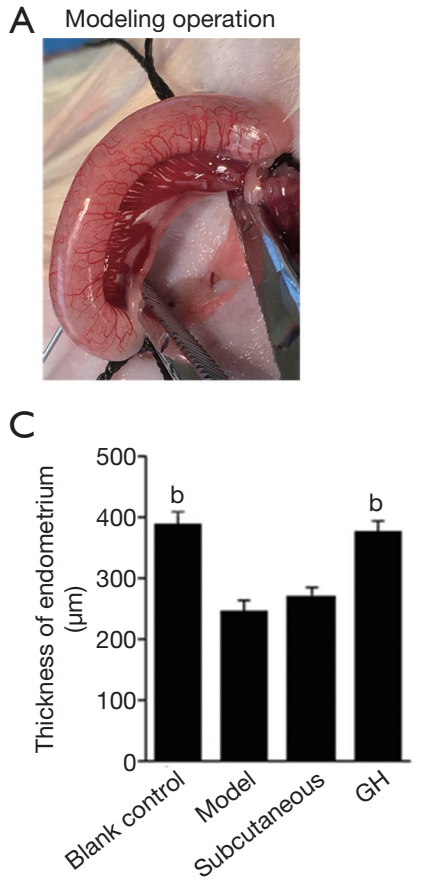

B

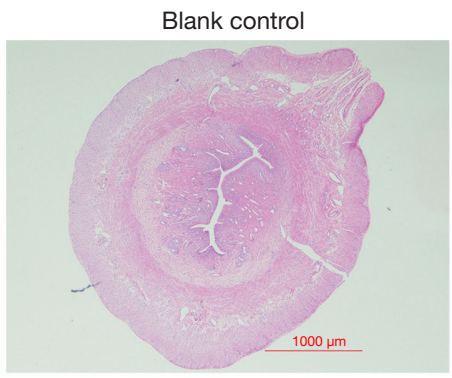

Subcutaneous

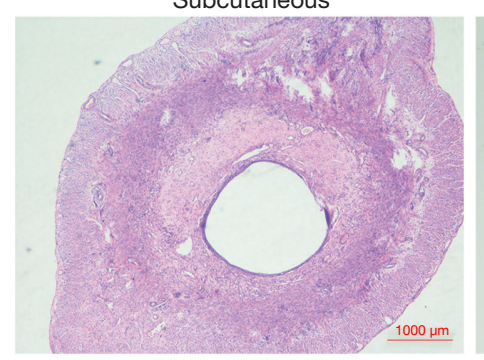

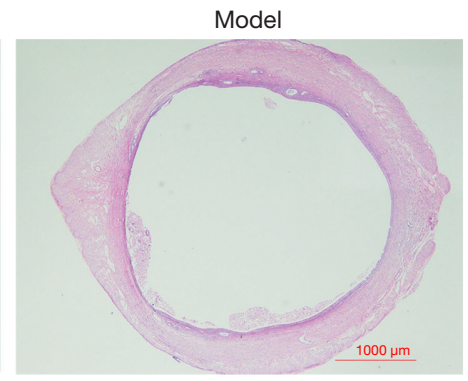

$\mathrm{GH}$



Figure 1 Endometria of the rats in the different groups $(\mathrm{HE}, \times 20)$. (A) The rats were intrauterine perfused with $95 \%$ ethanol during estrus to establish a thin endometrial model; (B) HE results of each group (scale bar 1:1,000); (C) results of endometrial thickness in each group, ${ }^{b} \mathrm{P}<0.01$. The intima thickness in the GH group and blank control group was increased compared with that in the model group. The intima thickness in the subcutaneous group was increased compared with that in the model group, but there was no significant difference. HE, hematoxylin and eosin; GH, growth hormone.

intima, base layer, and serosa were intact, and the glands and blood vessels were abundant. In the model group and subcutaneous group, the endometrium was thinner, uterine cavity was larger, and secretory glandular epithelial cells were sparsely arranged. In the GH group, the uterine structure was basically intact, and the intimal layer was thicker than that in the model group. The uterine cavity gap was significantly reduced compared with that in the model group; moreover, there was no obvious edema in the interstitial part. The intimal thickness in the GH group and blank control group were increased compared with that in the model group $(\mathrm{P}<0.01)$. The intima thickness in the subcutaneous group was increased compared with that in the model group, but there was no significant difference. There was no significant difference in the intimal thickness between the blank control group and the GH group (shown in Figure 1).

\section{IHC results}

Vimentin and cytokeratin are members of the cytoskeletal intermediate filament protein family. They are recognised as dynamic structures and participate in cell proliferation and differentiation. Cytokeratin is mainly found in rat endometrial secretory glandular epithelial cells and the luminal epithelial cytoplasm. Vimentin is mainly expressed in the rat endometrial mesenchyme cytoplasm. Brownyellow particle deposition was considered positive. The expression of cytokeratin and vimentin was increased in the GH group $(\mathrm{P}<0.01)$ and the blank control group $(\mathrm{P}<0.01)$. There was no significant difference between the blank control group and the GH group. The expression of cytokeratin and vimentin in the subcutaneous group was increased compared with that in the model group, but there was no significant difference (shown in Figure 2).

\section{Western blot results}

The expression of vimentin and cytokeratin in the GH group and blank control group were higher than that in the model group $(\mathrm{P}<0.01)$, but there was no significant difference between the blank control group and the GH 

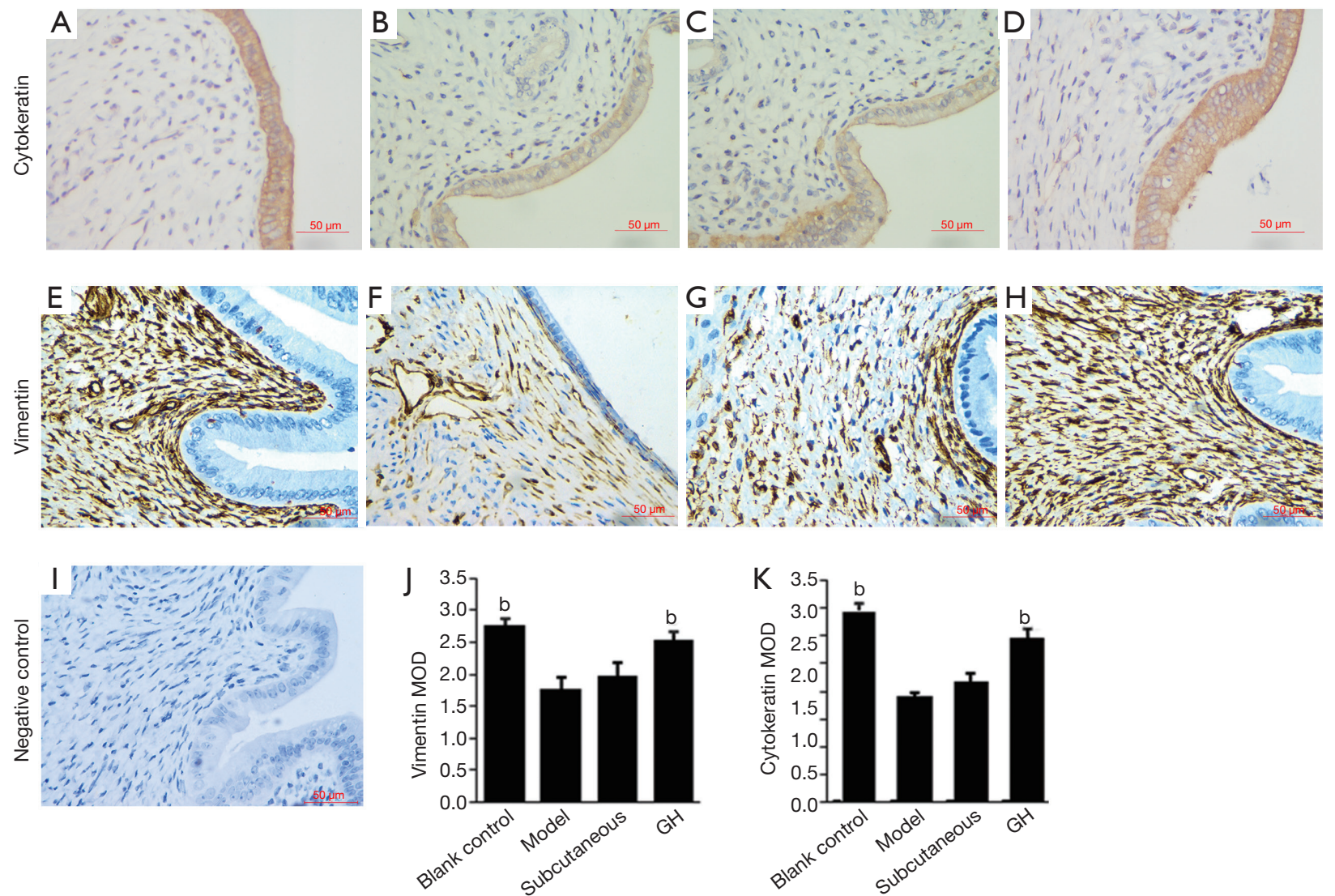

Figure 2 Expression of endometrial cytokeratin and vimentin in the different groups $(\times 400)$. Compared with the model group, ${ }^{b} \mathrm{P}<0.01$. (A-D) IHC results of cytokeratin in each group. Cytokeratin is mainly found in rat endometrial secretory glandular epithelial cells and the luminal epithelial cytoplasm and is an important protein for endometrial epithelial cell proliferation and cell anabolism (scale bar 1:50). (EH) IHC results of vimentin in each group. Vimentin is mainly expressed in the rat endometrial mesenchymal cytoplasm and is a marker protein for endometrial stromal cell proliferation and cell anabolism (scale bar 1:50). (I) Negative control (scale bar 1:50). (J,K) Comparison of cytokeratin and vimentin MOD values in each group. IHC, immunohistochemical; MOD, mean optical density.

group. The expression of vimentin and cytokeratin in the subcutaneous group was increased compared with that in the model group, but there was no significant difference (Figure 3).

\section{Discussion}

The American Society of Reproductive Medicine has indicated that with increasing endometrial thickness, clinical pregnancy and live birth or continued pregnancy rates significantly increase independent of patient age or embryo quality. A study involving more than 40,000 embryo transfers indicated that clinical pregnancy and the live birth rates decrease with each millimeter of reduction in endometrial thickness (11). However, to date, treatment of a thin endometrium has shown to be difficult.

This study explored the therapeutic effect of intrauterine perfusion of GH on thin endometria. We scientifically designed a randomized controlled trial to exclude other factors that may affect treatment, such as age, weight, and surgical injury. We successfully created model rats with thin endometria. In the model group, the intima thickness was significantly thinner than that in the control group. The expression levels of vimentin and cytokeratin were significantly reduced, which is consistent with the pathological features of a thin endometrium and the results reported by Miwa et al. (12).

An important role is played by $\mathrm{GH}$ in the reproductive 
A

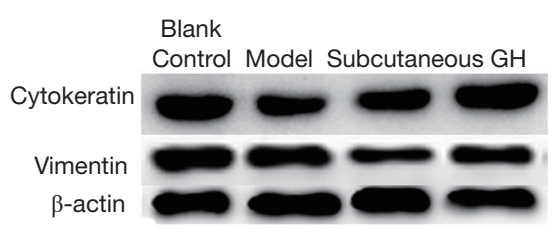

B

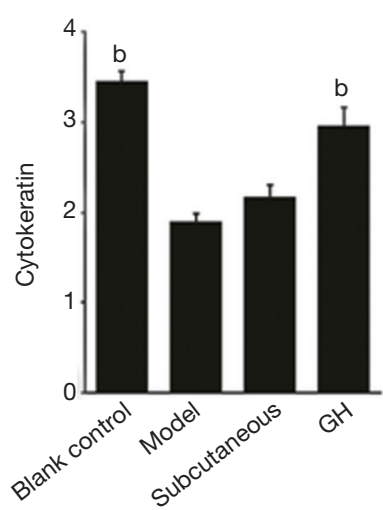

C

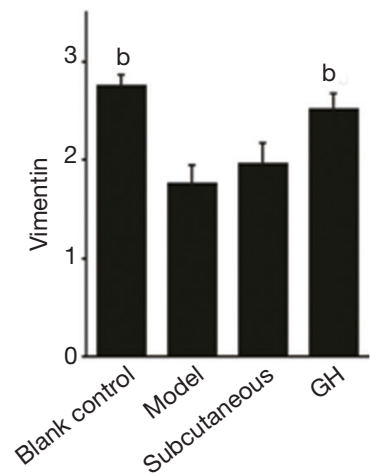

Figure 3 Protein expression of cytokeratin and vimentin in the endometria of rats in the different groups. (A) The expression of vimentin and cytokeratin in the GH group was higher than that in the model group. Compared with the model group, the expression of vimentin and cytokeratin was increased in the blank control group, but there was no significant difference between the blank control group and the GH group. The expression of vimentin and cytokeratin in the subcutaneous group was increased compared with that in the model group, but there was no significant difference. (B) Expression of cytokeratin with Western blot. (C) Expression of vimentin with western blot. GH, growth hormone. Compared with the model group, ${ }^{\text {b }} \mathrm{P}<0.01$.

process. Both human and rat endometrium have GH receptors. It can promote endometrial cell proliferation by binding IGF to the endometrium or the GH receptor (9). In our study, the thickness of the endometrium was significantly increased in the GH group compared with that in the model group, suggesting that the intrauterine infusion of GH played a positive role in the proliferation of endometrium. The main function of cytokeratin is to maintain the integrity and continuity of epithelial tissues, and the expression of cytokeratin level in cells is closely related to epithelial cell proliferation and differentiation. Vimentin is the most important intermediate fiber in mesenchymal cells, forming a cell scaffold along with microtubules and microfilaments. These proteins are all important markers for assessing cell proliferation (13). In our study, the expression of vimentin and cytokeratin in the GH group was significantly higher than that in the model group, suggesting that the proliferation and repair of the intima in the GH group was better than that in the model group.

Recently, intrauterine perfusion has become a new method in assisted reproductive treatment (14). It has been reported that peripheral perfusion of a peripheral blood mononuclear cell suspension can improve the implantation rate, live birth rate, and clinical pregnancy rate in patients with repeated implantation failure (15). For the first time, Gleicher et al. (16) used colony-stimulating factor to treat thin endometrium and achieved a favorable outcome. A prospective study indicated that intrauterine injection of human chorionic gonadotropin prior to embryo transfer significantly increased the implantation rate and pregnancy rate in IVF/intracytoplasmic sperm injection (ICSI) (17). Currently, research concerning the intrauterine infusion of $\mathrm{GH}$ for the treatment of a thin endometrium in rats is lacking. Therefore, we first proposed the intrauterine infusion of $\mathrm{GH}$ for the treatment of rats with thin endometrium. In our study, we added the subcutaneous group for comparison of therapeutic effect with the GH group. The thickness of the endometrium and the expression of vimentin and cytokeratin in the GH group were significantly higher than those in the subcutaneous injection group, suggesting that the therapeutic effect of uterine infusion of $\mathrm{GH}$ is better.

\section{Conclusions}

In summary, based on the successful construction of model rats, intrauterine infusion of GH significantly increased the thickness of endometrium. The experiments showed that the intrauterine perfusion of GH may be a new therapeutic approach for the treatment of a thin endometrium and further research is warranted.

\section{Acknowledgments}

Funding: This work was supported by the Introduced Talent Fund of Yi Jishan Hospital (KY25220349). 


\section{Footnote}

Reporting Checklist: The authors have completed the ARRIVE reporting checklist. Available at https://dx.doi. org/10.21037/atm-21-3583

Data Sharing Statement: Available at https://dx.doi. org/10.21037/atm-21-3583

Conflicts of Interest: All authors have completed the ICMJE uniform disclosure form (available at https://dx.doi. org/10.21037/atm-21-3583). The authors have no conflicts of interest to declare.

Ethical Statement: The authors are accountable for all aspects of the work in ensuring that questions related to the accuracy or integrity of any part of the work are appropriately investigated and resolved. Experiments were performed under a project license (No.: LLSC-2019-001) granted by the First Affiliated Yijishan Hospital of Wannan Medical College Ethics Committee. All animal experiments are carried out in accordance with the National Institutes of Health Guide for the Care and Use of Laboratory animals.

Open Access Statement: This is an Open Access article distributed in accordance with the Creative Commons Attribution-NonCommercial-NoDerivs 4.0 International License (CC BY-NC-ND 4.0), which permits the noncommercial replication and distribution of the article with the strict proviso that no changes or edits are made and the original work is properly cited (including links to both the formal publication through the relevant DOI and the license). See: https://creativecommons.org/licenses/by-nc-nd/4.0/.

\section{References}

1. Kasius A, Smit JG, Torrance HL, et al. Endometrial thickness and pregnancy rates after IVF: a systematic review and meta-analysis. Hum Reprod Update 2014;20:530-41.

2. Kunicki M, Łukaszuk K, Woclawek-Potocka I, et al. Evaluation of granulocyte colony-stimulating factor effects on treatment-resistant thin endometrium in women undergoing in vitro fertilization. Biomed Res Int 2014;2014:913235.

3. Kovacs P, Matyas S, Boda K, et al. The effect of endometrial thickness on IVF/ICSI outcome. Hum Reprod 2003;18:2337-41.
4. von Wolff M, Fäh M, Roumet M, et al. Thin Endometrium Is Also Associated With Lower Clinical Pregnancy Rate in Unstimulated Menstrual Cycles: A Study Based on Natural Cycle IVF. Front Endocrinol (Lausanne) 2018;9:776.

5. Chen Y, Liu L, Luo Y, et al. Effects of Aspirin and Intrauterine Balloon on Endometrial Repair and Reproductive Prognosis in Patients with Severe Intrauterine Adhesion: A Prospective Cohort Study. Biomed Res Int 2017;2017:8526104.

6. Zheng SX, Wang J, Wang XL, et al. Feasibility analysis of treating severe intrauterine adhesions by transplanting menstrual blood-derived stem cells. Int J Mol Med 2018;41:2201-12.

7. Sbracia M, Scarpellini F, Poverini R, et al. Immunohistochemical localization of the growth hormone in human endometrium and decidua. Am J Reprod Immunol 2004;51:112-6.

8. Berger C, Boggavarapu NR, Menezes J, et al. Effects of ulipristal acetate on human embryo attachment and endometrial cell gene expression in an in vitro co-culture system. Hum Reprod 2015;30:800-11.

9. Cui N, Li AM, Luo ZY, et al. Effects of growth hormone on pregnancy rates of patients with thin endometrium. J Endocrinol Invest 2019;42:27-35.

10. Xu Y, Zhao G, Miao J, et al. Changes in vimentin and vascular endothelial growth factor expression in a rat model of thin endometrium established by $95 \%$ ethanol. Chinese Journal of Tissue Engineering Research 2016;20:718-22.

11. Liu KE, Hartman M, Hartman A, et al. The impact of a thin endometrial lining on fresh and frozen-thaw IVF outcomes: an analysis of over 40000 embryo transfers. Hum Reprod 2018;33:1883-8.

12. Miwa I, Tamura H, Takasaki A, et al. Pathophysiologic features of "thin" endometrium. Fertil Steril 2009;91:9981004.

13. Wolthers T, Hoffman DM, Nugent AG, et al. Oral estrogen antagonizes the metabolic actions of growth hormone in growth hormone-deficient women. Am J Physiol Endocrinol Metab 2001;281:E1191-6.

14. Huang P, Wei L, Li X. A study of intrauterine infusion of human chorionic gonadotropin (hCG) before frozenthawed embryo transfer after two or more implantation failures. Gynecol Endocrinol 2017;33:67-9.

15. Yoshioka S, Fujiwara H, Nakayama T, et al. Intrauterine administration of autologous peripheral blood mononuclear cells promotes implantation rates in patients with repeated failure of IVF-embryo transfer. Hum 
Page 8 of 8

Reprod 2006;21:3290-4.

16. Gleicher N, Vidali A, Barad DH. Successful treatment of unresponsive thin endometrium. Fertil Steril 2011;95:2123.e13-7.

17. Mansour R, Tawab N, Kamal O, et al. Intrauterine

Cite this article as: $\mathrm{Li}$ W, Cao Z, Yu X, Hu W. Effect of growth hormone on thin endometrium via intrauterine infusion. Ann Transl Med 2021;9(16):1325. doi: 10.21037/atm-21-3583
Li et al. Effect of growth hormone on thin endometrium

injection of human chorionic gonadotropin before embryo transfer significantly improves the implantation and pregnancy rates in in vitro fertilization/intracytoplasmic sperm injection: a prospective randomized study. Fertil Steril 2011;96:1370-1374.e1. 